\title{
Spatial damping of propagating kink waves due to mode coupling
}

\author{
D. J. Pascoe, A. W. Hood, I. De Moortel, and A. N. Wright
}

\author{
School of Mathematics and Statistics, University of St Andrews, St Andrews, KY16 9SS, UK \\ e-mail: dpascoe@mcs.st-and.ac.uk
}

Received 30 August 2011 / Accepted 12 December 2011

\begin{abstract}
Aims. We investigate the damping process for propagating transverse velocity oscillations, observed to be ubiquitous in the solar corona, due to mode coupling.

Methods. We perform 3D numerical simulations of footpoint-driven transverse waves propagating in a low $\beta$ coronal plasma with a cylindrical density structure. Mode coupling in an inhomogeneous layer leads to the coupling of the kink mode to the Alfvén mode, observed as the decay of the transverse kink oscillations.

Results. We consider the spatial damping profile and find a Gaussian damping profile of the form $\exp \left(-z^{2} / L_{\mathrm{g}}^{2}\right)$ to be the most congruent with our numerical data, rather than the exponential damping profile of the form $\exp \left(-z / L_{\mathrm{d}}\right)$ used in normal mode analysis. Our results highlight that the nature of the driver itself will have a substantial influence on observed propagating kink waves.

Conclusions. Our study suggests that this modified damping profile should be taken into account when using coronal seismology to infer local plasma properties from observed damped oscillations.
\end{abstract}

Key words. Sun: corona - magnetohydrodynamics (MHD) - Sun: atmosphere - Sun: magnetic topology - waves

\section{Introduction}

Standing kink oscillations of coronal loops have attracted attention as a possible diagnostic tool for the solar corona since the observations of post-flare loops by the TRACE satellite (e.g., Aschwanden et al. 1999; Nakariakov et al. 1999). More recently, there has been interest in propagating kink waves, following Tomczyk et al. (2007) and Tomczyk \& McIntosh (2009) who used the ground-based coronagraph CoMP to observe spatially and temporally ubiquitous propagating transverse velocity oscillations with periods of about 5 min. Tomczyk \& McIntosh (2009) reported strong damping of these propagating waves which was interpreted by Pascoe et al. (2010) in terms of coupling between the kink and Alfvén modes.

For the case of standing kink modes in a coronal loop being damped by resonant absorption, the kink eigenmode has a spatial structure determined by the loop parameters and the harmonic order of the mode. The global kink mode, for example, has order $N=1$ and a wavelength $\lambda=2 L / N$ for a loop of length $L$. The standing mode amplitude varies as a function of position along the loop. It is therefore instructive to consider a fixed point along the loop, such as the anti-node of displacement, and then determine the oscillation amplitude as a function of time. For standing kink modes, an exponential damping envelope is obtained (e.g., Ruderman \& Roberts 2002) of the form

$A(t)=A_{0} \exp (-t / \tau)$

Here the damping time $\tau$ depends on the period of oscillation $P$ and the loop parameters as

$\frac{\tau}{P}=C \frac{a}{l} \frac{\rho_{0}+\rho_{\mathrm{e}}}{\rho_{0}-\rho_{\mathrm{e}}}$,

where $a$ is the loop radius, $l$ is the inhomogeneous layer thickness, and $\rho_{0}$ and $\rho_{\mathrm{e}}$ are the internal and external mass densities, respectively. The constant $C$ depends upon the chosen density profile in the inhomogeneous layer, e.g. for a linear density profile $C=(2 / \pi)^{2}$ (see e.g., Hollweg \& Yang 1988; Goossens et al. 1992). This equation was derived using the thin tube, thin boundary (TTTB) approximation. The assumption of a thin boundary was relaxed by Van Doorsselaere et al. (2004) who performed a numerical investigation for thick inhomogeneous layers.

Terradas et al. (2006) performed numerical simulations for standing kink modes in straight line-tied coronal loops with a thick inhomogeneous layer. The standing kink modes are excited by a planar pulse and, after an initial transitory period, undergo exponential damping by resonant absorption as given by Eqs. (1) and (2).

For a propagating kink wavepacket in an inhomogeneous loop, mode coupling will cause the kink oscillations to decay. The mode coupling condition is satisfied where $\omega=$ $V_{\mathrm{A}}(\boldsymbol{r}) k_{z}$, where $\omega$ is the dominant (angular) frequency of the kink wavepacket, $V_{\mathrm{A}}(\boldsymbol{r})$ is the local Alfvén speed and $k_{z}$ is the local longitudinal wavenumber (Pascoe et al. 2011a). If the wavepacket propagates with group speed $V_{\mathrm{g}}=\partial \omega / \partial k$, then at a time $t$ the distance it has propagated along the loop is $z=V_{\mathrm{g}} t$. We can, therefore, consider the damping of the wavepacket roughly as a function of distance as

$A(z)=A_{0} \exp \left(-z / L_{\mathrm{d}}\right)$

where $L_{\mathrm{d}}=V_{\mathrm{g}} \tau$ is the damping length (Pascoe et al. 2010). Since $\tau \propto P \propto 1 / f$, the damping length will depend on the frequency of the oscillation. Terradas et al. (2010) considered the frequency-dependence of the damping length scale in detail, and Verth et al. (2010) found evidence of the effect of mode coupling acting as a low-pass filter in CoMP data.

These results strictly apply to the situation where all fields are varying as $\exp i\left(k_{z} z-\omega t\right)$, but provide a useful guide for the decay length of wavepackets and other situations where there is not a single frequency or wavenumber such as initial value problems or systems with a broadband frequency driver. 
In this paper, we focus on the damping profile as a function of height for propagating kink waves driven by harmonic footpoint motions. We find that for propagating modes, the exponential damping profile does not provide an adequate account of the decay at low heights.

\section{Model}

The initial equilibrium we consider is the same as in Pascoe et al. (2010). We consider a straight, uniform magnetic field in the $z$ direction. We choose the plasma $\beta=0.001$ to be small, appropriate for the solar corona. Our density profile describes a cylindrical tube aligned with the $z$ axis. We define a cylindrical core region with radius $r \leq b$, surrounded by an inhomogeneous layer $b<r \leq a$. The density is $\rho_{0}$ in the core region and $\rho_{\mathrm{e}}$ in the external region $(r>a)$. In between is the inhomogeneous layer of width $l=a-b$, where the density varies linearly from $\rho_{0}$ to $\rho_{\mathrm{e}}$. We choose a density contrast $\rho_{0} / \rho_{\mathrm{e}}=2$ and inhomogeneous layer thickness $l / a=0.5$. The Alfvén speed varies from $C_{\mathrm{A} 0}$ in the core region to $C_{\mathrm{Ae}}$ in the external region.

Rather than simulating a finite wavetrain as in Pascoe et al. (2010), the driver is chosen to simulate continuous harmonic footpoint motions displacing the tube axis. The driving condition is applied to the lower $z$ boundary and prescribes the $x$ and $y$ components of velocity,

$\boldsymbol{v}=\sin (\omega t) \boldsymbol{u}, \boldsymbol{u}(x, y)=\left(u_{x}, u_{y}, 0\right)$

where $\omega$ is the (constant) angular frequency of the driver and where the spatial dependence $\boldsymbol{u}(x, y)$ has the same dipole form as in Pascoe et al. (2010) (see their Eq. (4) and discussion in text). We choose the maximum value of the perturbation $u / C_{\mathrm{Ae}}=0.002$ to be small in order to avoid non-linear effects.

Modelling an exact kink normal mode requires a driver with an azimuthal velocity profile that is not just discontinuous but actually singular. Instead a reasonable compromise could be to consider an azimuthal velocity with a step function (finite) discontinuity. Such a driver (as in Terradas et al. 2010) would excite mainly the kink normal mode with only a very small contribution from other disturbances. When using this particular driver in a thin flux tube with a narrow inhomogeneous layer (TTTB approximation), the exponential damping of Terradas et al. (2010) is to be expected. However, it is unlikely that the Sun will produce a driving velocity pattern that is discontinuous so we mainly consider a smooth (i.e., without discontinuities) driver.

The simulations are performed using the MHD code LARE3D (Arber et al. 2001). The numerical domain is much larger in the $z$ direction than in $x$ or $y$ in order to accommodate the continuously driven propagating wavepacket. However, the resolution is higher in the $x$ and $y$ directions in order to resolve the activity in the inhomogeneous layer for as long as possible, particularly when phase mixing of the Alfvén mode takes place. Typical values used are $400 \times 400 \times 400$ grid points for a numerical domain of $6 \times 6 \times 150 \mathrm{Mm}(a=1 \mathrm{Mm})$. The boundary conditions are periodic in the $x$ and $y$ directions, and are placed sufficiently far from the flux tube to not affect the results. All simulations were run without resistivity.

\section{Results}

The transverse velocity perturbations excited at the lower boundary propagate along the magnetic field. The simulation ends before perturbations reach the upper boundary, to avoid unwanted reflections.
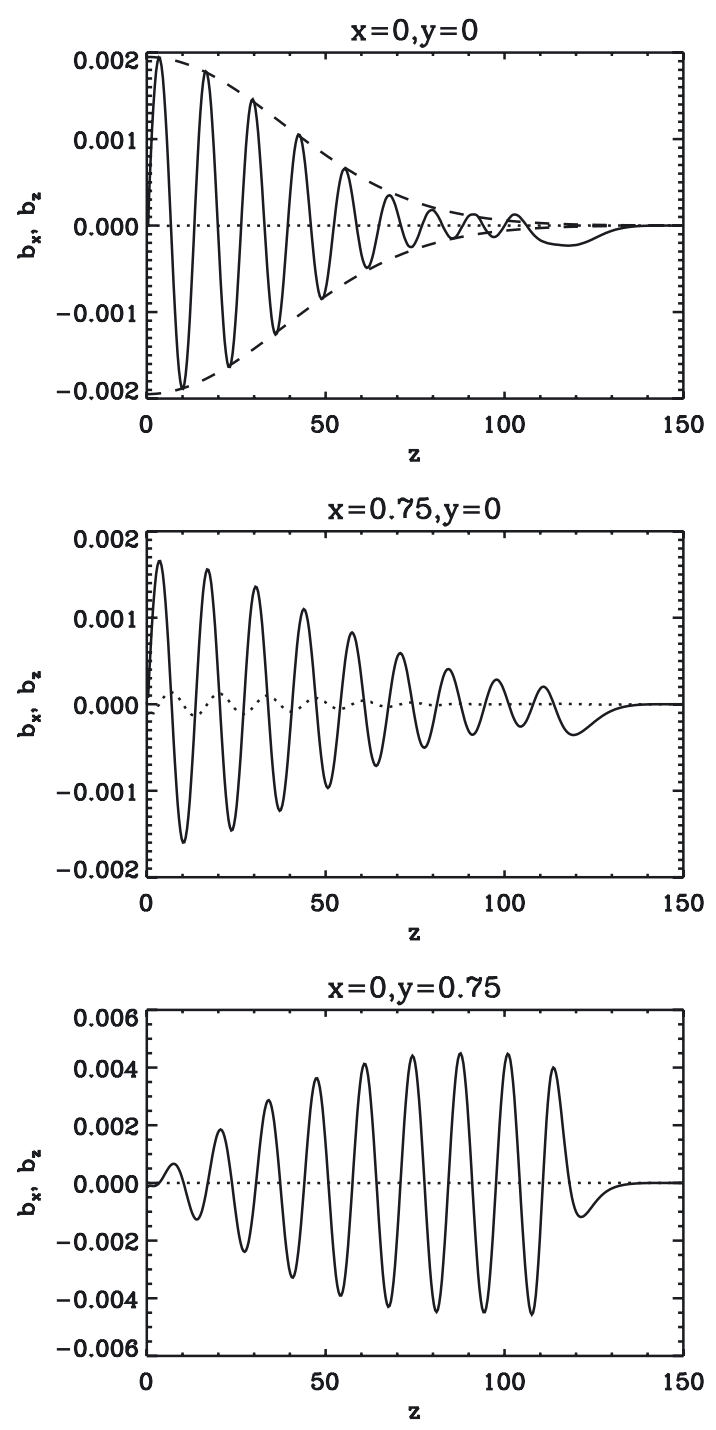

Fig. 1. Magnetic field components as a function of height for a simulation driven with a single harmonic frequency. The solid lines and dotted lines represent $b_{x}$ and $b_{z}$, respectively. The top panel shows the kink mode along the loop axis. The middle panel shows the kink mode inside the inhomogeneous layer. The bottom panel shows the Alfvén mode in the inhomogeneous layer. (Note that $b_{z}$ has nodes at the $(x, y)$ locations in the top and bottom panels.)

Figure 1 shows the resultant magnetic field components, each as a function of height (or propagation distance) $z$, at several locations in the numerical domain. The upper panel shows the perturbations to the magnetic field $b_{x}$ (solid line) and $b_{z}$ (dotted line) at the loop axis, where $\boldsymbol{b}(t)=\boldsymbol{B}(t)-\boldsymbol{B}(t=0)$. The component $b_{z}$ has small fluctuations indicating an almost incompressible (kink) mode, and $b_{x}$ varies harmonically according to the driven footpoint oscillation which propagates upwards and undergoes damping due to mode coupling. The dashed line is a damping profile with a Gaussian form

$A(z)=A_{0} \exp \left(-z^{2} / L_{\mathrm{g}}^{2}\right)$

where $L_{\mathrm{g}}$ is empirically determined by fitting. Although $L_{\mathrm{g}}$ has been chosen to give the best fit, the form of the Gaussian profile seems much more congruent with the numerical data than the exponential envelope found in the modelling of Terradas et al. (2010) since the radial profile of a smooth driver in a flux tube with a wide inhomogeneous layer cannot be represented by a single eigenmode. It is clear that an exponential 
D. J. Pascoe et al.: Spatial damping of propagating kink waves

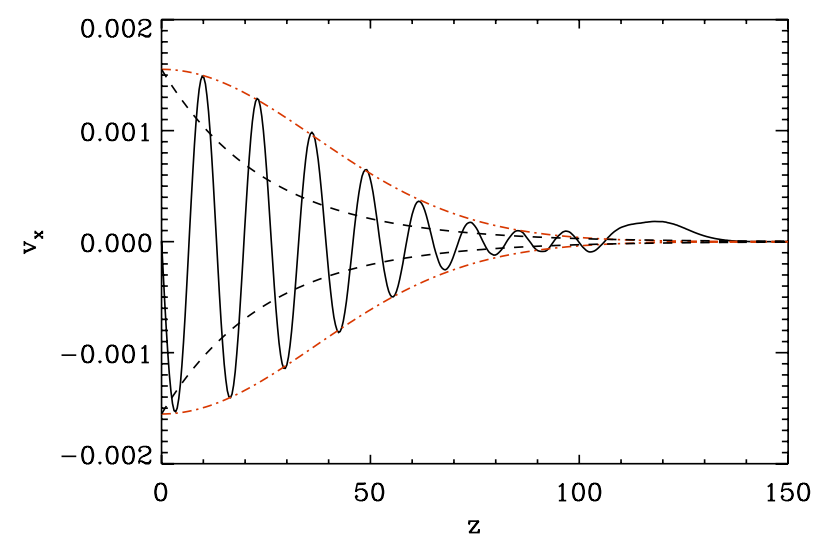

Fig. 2. Transverse velocity component $v_{x}$ as a function of height at the loop axis. The dot-dashed (red) line represents a Gaussian envelope of the form given in Eq. (5). The dashed line is the exponential decay given by Eqs. (2) and (3).

damping profile, as demonstrated by standing modes, would not provide an adequate fit to our results. The Gaussian damping profile produces an excellent fit to the data over all values of $z$, except for the expected disparity at the leading edge (see e.g., Hood et al. 2005, for the case of damping of wavetrains by phase mixing).

For this particular simulation the boundary was driven with $\omega=0.377 \mathrm{~s}^{-1}$. Our simulation was repeated for several driver frequencies, each showing the same Gaussian damping profile, with its own particular value of $L_{\mathrm{g}}$ which decreases with frequency as $L_{\mathrm{g}} \propto 1 / f$.

The middle panel of Fig. 1 shows the magnetic field components as a function of $z$ at $y=0$ and at the centre of the inhomogeneous layer $(x=0.75)$. This region is close to the (radial) antinode in $b_{z}$. Note that our driver (Eq. (4)) is compressible in the transition layer and the amount of compression will influence the magnitude of $b_{z}$. The lower panel shows the magnetic field components again at the centre of the inhomogeneous layer but at $x=0$ (where $b_{z}$ has a radial node). This is also the location of the antinode for the $m=1$ Alfvén mode, represented by $b_{x}$, which grows in $z$ as energy is transferred to the Alfvén mode from the kink mode. It is interesting to note that a similar growth in Alfvén wave amplitude was found in the simulations and modelling of Mann \& Wright (1995) and Mann et al. (1997).

Figure 2 shows the transverse velocity component $v_{x}$ as a function of height at the loop axis with two spatial damping profiles added. The dot-dashed (red) line represents a Gaussian envelope of the form given in Eq. (5). The dashed line is the exponential decay given by Eqs. (2) and (3) with $V_{\mathrm{g}}=C_{k}=$ $\sqrt{2 /\left(1+\rho_{\mathrm{e}} / \rho_{0}\right)} C_{\mathrm{A} 0}$ and $C=(2 / \pi)^{2}$ as in, e.g., Terradas et al. (2010). This formula, which is commonly used and is based upon the thin flux tube and thin boundary layer approximations, gives an exponential damping length of $L_{\mathrm{d}} \approx 25 \mathrm{Mm}$. Our simulations indicate a different (Gaussian) envelope is more appropriate and has a larger length scale of $L_{\mathrm{g}} \approx 52 \mathrm{Mm}$. These differences must be accounted for when interpreting data and simulations.

\subsection{Numerical simulation in cylindrical coordinates}

In order to confirm our results in the absence of an analytical treatment, we perform the same experiment with an independent numerical code in which perturbations are proportional to $\exp (\mathrm{i} \theta)$. This alternative Lax-Wendroff code differs in using a
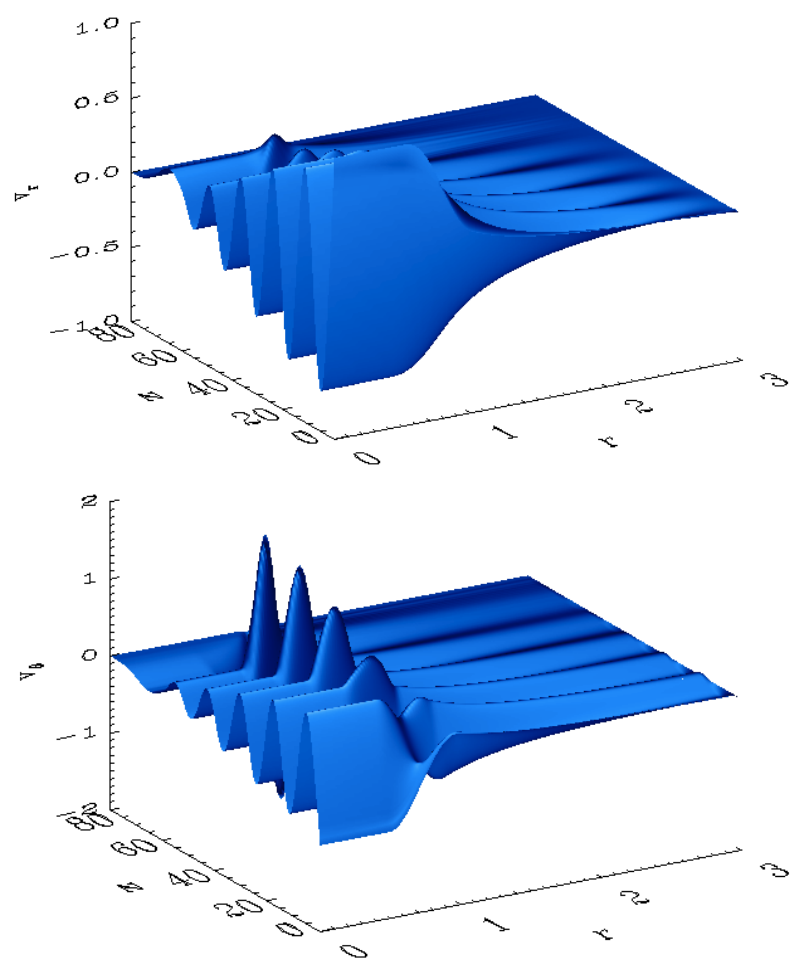

Fig. 3. Snapshot of radial velocity $v_{r}(t o p)$ and $v_{\theta}$ (bottom) as a function of $r$ and $z$ for a kink wave propagating in a cylindrical coronal loop with an inhomogeneous layer $(0.5<r \leq 1)$. The velocity components in the core $(r \leq 0.5)$ are damped as the energy is transferred by mode coupling to azimuthal Alfvén oscillations in the inhomogeneous layer.

cylindrical rather than Cartesian coordinate system. It also solves the linear MHD equations, rather than LARE3D which solves the full nonlinear MHD equations although we consider small amplitude perturbations to approximate the linear regime. The same initial equilibrium, driver and boundary conditions are used as in the results discussed above.

Figure 3 shows snapshots of the radial and azimuthal velocities at the end of the simulation. Both velocity components show damping with increasing height $z$ in the core region $r \leq 0.5$. The lower panel clearly shows the growth of the Alfvén mode, $v_{\theta}$, inside the inhomogeneous shell.

Figure 4 shows a plot of $v_{r}$ (top) and $b_{z}$ (bottom) as a function of height $z$ at the centre of the inhomogeneous layer (i.e. where the condition for mode coupling is satisfied). The dashed line is a Gaussian envelope and is in excellent agreement with the calculated damping rate, except again for the expected disparity at the leading edge (e.g., Hood et al. 2005).

The top panel of Fig. 5 shows the location of the zeroes of $v_{r}$ (crosses) and $b_{z}$ (diamonds) for the oscillations in Fig. 4. The zeroes for $b_{z}$ have been shifted to be in phase with those for $v_{r}$. The dashed (red) line shows the predicted locations based on the driving period $P$ and the kink speed $C_{k}$. In the long-wavelength limit $k a \ll 1$, the kink mode will have wavelength $\lambda \approx C_{k} P$ so the zeroes are separated by $\lambda / 2$. The zeroes for $b_{z}$ are in good agreement with the predictions based on the kink mode. The dot-dashed (black) line shows the predicted locations of the zeroes based on the Alfvén speed $C_{\mathrm{A} 0}$. The zeroes of $v_{r}$ initially follow the estimate based on the kink speed, but later switch $(n>4)$ to follow more closely the estimate based on the internal Alfvén speed. This crossover provides more support for the interpretation of propagating transverse velocity perturbations as 

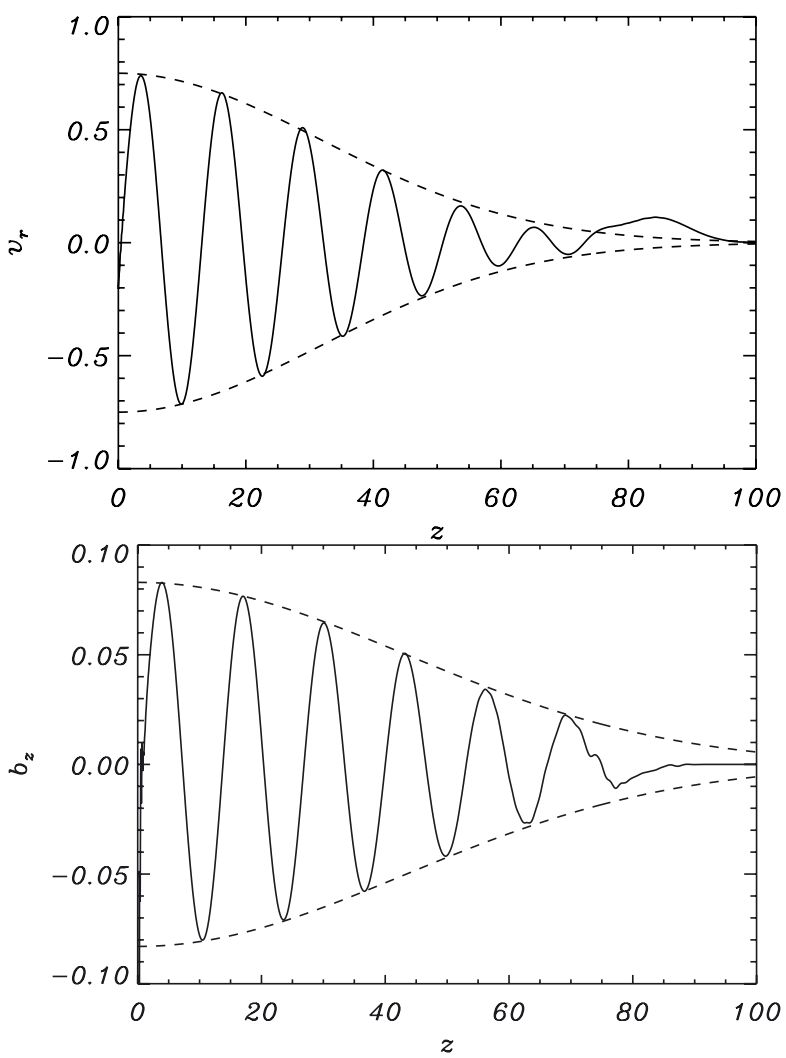

Fig. 4. Radial velocity $v_{r}$ (top) and longitudinal magnetic field $b_{z}$ (bottom) as a function of height $z$ at the centre of the inhomogeneous layer. The dashed lines represent a Gaussian envelope of the form given in Eq. (5).

a genuine coupling of the kink and Alfvén modes (Pascoe et al. 2010).

The bottom panel of Fig. 5 shows the natural logarithm of the amplitude of the $v_{r}$ signal as a function of height. The dashed line represents a quadratic fit, supporting the Gaussian envelope in Eq. (5).

\section{Discussion}

We have considered the propagation of harmonic transverse velocity perturbations through a low $\beta$ plasma with cylindrical density structure. The transfer of energy from transverse kink oscillations to azimuthal Alfvén motions, due to mode coupling, is observed as a decay of the initial kink disturbance. For a broadband driver, each Fourier component can be considered to have a damping length scale $L_{\mathrm{d}}=V_{\mathrm{g}} \tau$, where $\tau \propto P \propto 1 / f$. Higher frequency components will therefore be damped over shorter propagation distances than lower frequency components. In this way mode coupling acts as a low-pass filter, as noted by Terradas et al. (2010) and Verth et al. (2010).

Pascoe et al. (2010) showed that normal mode calculations can be a useful indicator of the behaviour of propagating wavetrains when the spatial and temporal scales match (see also Terradas et al. 2010). In this paper, we have demonstrated that the damping profile of propagating transverse velocity perturbations in a flux tube with a wide inhomogeneous layer differs from the exponential profile of standing or normal modes in $z$, and that a Gaussian damping profile is most congruent with our numerical data. However, for a flux tube with a narrow inhomogeneous layer (TTTB), the result of Terradas et al. (2010) should be recovered in the case of a driver with a singular azimuthal velocity
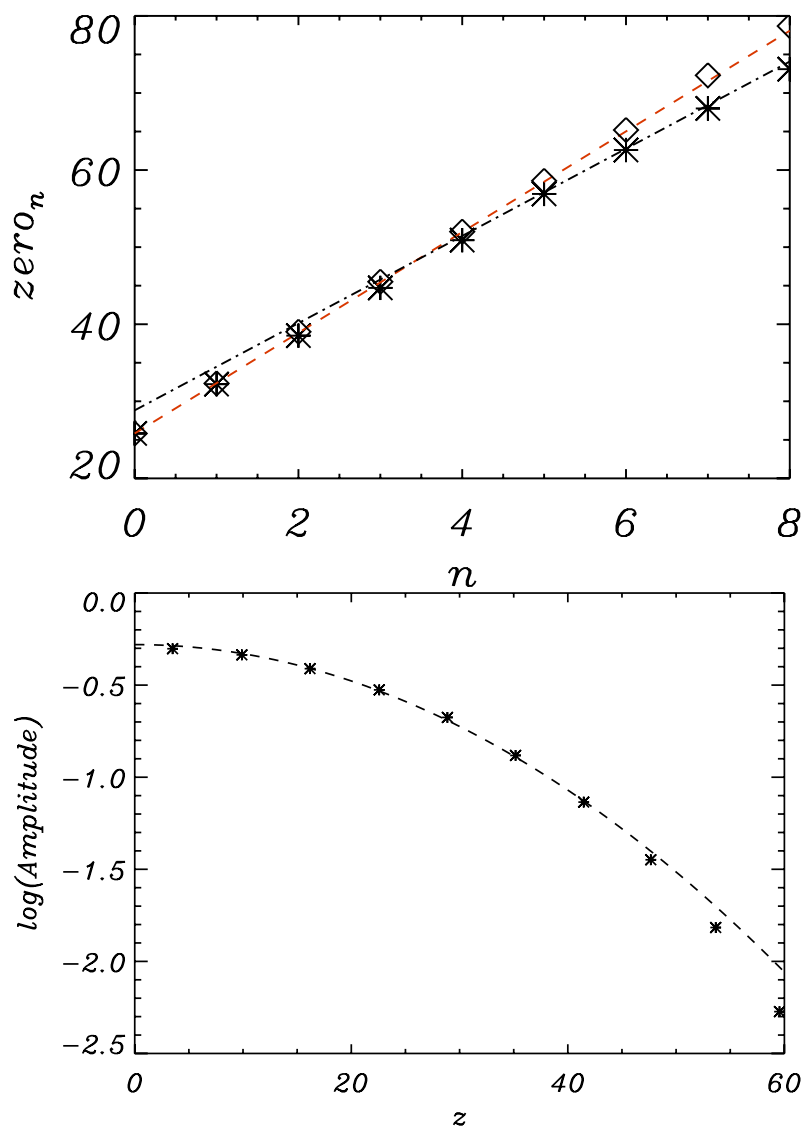

Fig. 5. The top panel shows the location of the zeroes of $v_{r}$ (crosses) and $b_{z}$ (diamonds) for the oscillations in Fig. 4. The zeroes for $b_{z}$ have been shifted to be in phase with those for $v_{r}$. The dashed (red) and dotdashed (black) lines represent the predicted values for the kink mode and Alfvén mode, respectively. The bottom panel shows the natural logarithm of the amplitude of the $v_{r}$ signal as a function of height. The dashed line indicates Gaussian nature of the spatial damping profile.

profile of the form $1 /(r-R)$, where $R$ is the radius at which the kink speed equals the local Alfvén speed. This is demonstrated in Fig. 6, where the top panel shows the logarithmic amplitude of the radial velocity perturbations at the axis of a flux tube with a narrow inhomogeneous layer with $l / a=0.1$. These perturbations were generated by a driver with a step function discontinuity in the radial profile of the azimuthal velocity. The bottom panel, on the other hand, shows the simulation result using a smooth driving profile and a wide inhomogeneous layer $l / a=$ $2 / 3$. In both panels the dashed line corresponds to an exponential damping profile, whereas the dot-dashed line corresponds to a Gaussian damping profile. It is clear that for the top panel (TTTB model) the exponential decay gives an excellent fit after the first few wavelengths, demonstrating that in this limit we do indeed recover the results of Terradas et al. (2010). For a smooth (continuous) driver with a wide inhomogeneous layer, the damping profile is mainly Gaussian apart from at large heights where the exponential profile is recovered. However, in the TTTB regime (top panel) the damping is very weak even at large heights. The observations have shown strong in situ attenuation of the transverse velocity perturbations (Tomczyk \& McIntosh 2009) and hence, the strong damping achieved in our wide layer simulations (bottom panel) seems more representative. Accordingly, care should be taken when using the spatial damping length in coronal seismology applications. For example, when strong damping is observed, our simulations show that fitting to an 

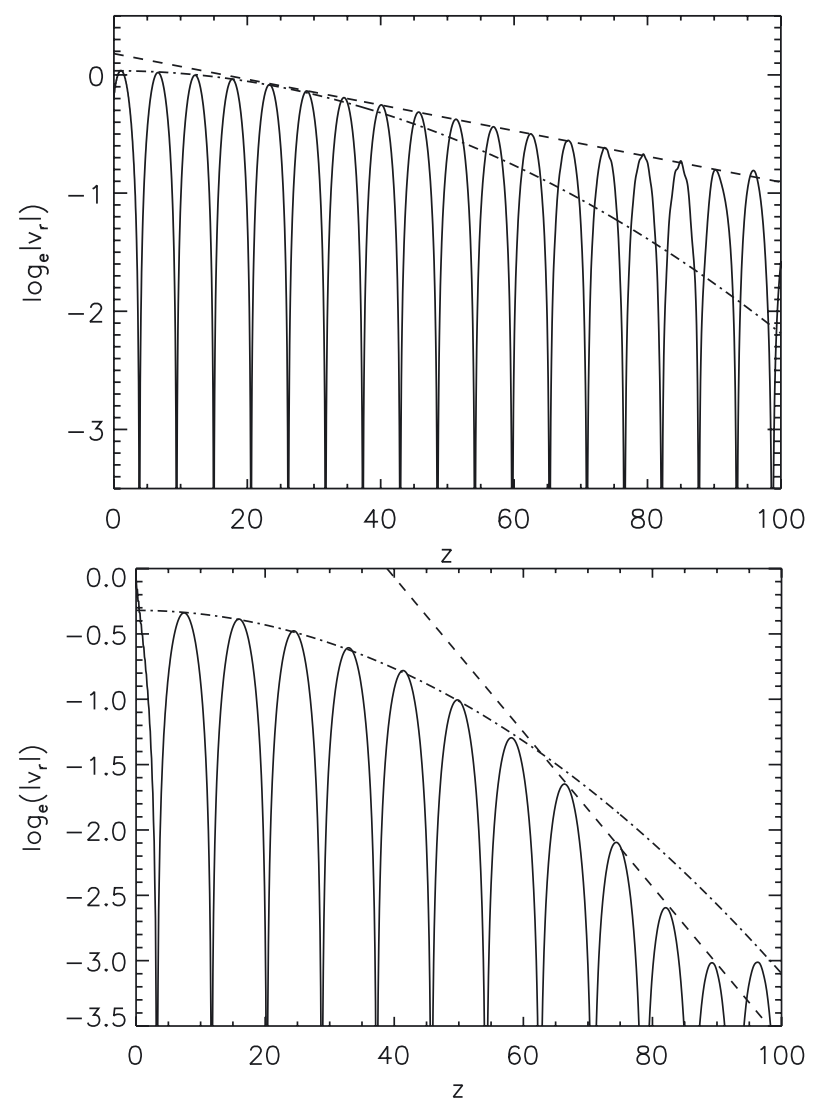

Fig. 6. Logarithmic amplitude of the radial velocity perturbations at the axis of a flux tube for a narrow inhomogeneous layer with $l / a=0.1$, using a driver with a radially discontinuous azimuthal component (top) and for a wide inhomogeneous layer $l / a=2 / 3$, using a smooth driver (bottom). The dashed and dot-dashed lines correspond to exponential and Gaussian spatial damping profiles, respectively.

exponential damping rate low in the corona would underestimate the mode coupling rate and could infer, say, less density structuring than is actually present.

If we consider the excitation of a standing kink mode in a coronal loop with an inhomogeneous layer, if the exact kink eigenmode is applied, the kink mode will decay exponentially as resonant absorption transfers the wave energy to an Alfvén mode. If the exact eigenmode is not applied, for example the kink mode is excited by some external perturbation, then there will be an initial transitory period, of order $2 L / C_{\mathrm{A} 0}$, as the kink mode is established. After this time, the standing kink mode will decay in the usual way. When we now consider propagating kink modes, it is convenient to substitute distance for time $\left(z=V_{\mathrm{g}} t\right)$ and consider a damping length $L_{\mathrm{d}}$ rather than damping time $\tau$. However, the disparity between the exponential and Gaussian damping profiles at low $z$ presented here ought not to be confused with the transient that is present at early times in the case of the standing mode. For a propagating mode, the transient behaviour applies to the first (and last) driving cycle as discussed by Hood et al. (2005). For an established, continuous driver, the Gaussian damping profile will apply for mode coupling at all times. For a broadband driver, each frequency will follow the Gaussian profile with its own particular damping length (Pascoe et al. 2011b).

Pascoe et al. (2010) found that the normal mode analysis for resonant absorption was a useful guide to the behaviour of propagating kink wavepackets with regard to their characteristic damping length $L_{\mathrm{d}}$. Pascoe et al. (2011a) noted that the method of mode coupling for propagating kink wavepackets differs from resonant absorption of standing modes in that there is no resonant singularity in this process and there is no harmonic driving frequency. The result of this paper supports the claim that normal mode analysis provides a useful guide to the behaviour of propagating wavepackets but a more considered approach is required if propagating waves are intended to be used as an accurate diagnostic for MHD coronal seismology. However, even for the described Gaussian damping profile of the driven transverse velocity perturbations, mode coupling will still act as a frequency filter with the higher frequencies damped near the loop footpoints and only the lower frequency modes propagating further along the loops. If the wrong spatial envelope for damped kink modes is used when interpreting data, it is inevitable that any inferences made will be questionable and/or misleading.

The Gaussian rather than exponential spatial damping profile arises because we do not apply a pure kink mode driver and have a wide inhomogeneous layer. A full analytical treatment and parametric study of the dependence of the damping length scale $L_{\mathrm{g}}$ on driver frequency and density structuring will be presented in a forthcoming paper. Results so far indicate that if the transition layer is very narrow, then the exponential damping profile of Terradas et al. (2010) is a good approximation for nearly all heights. If the transition layer is wider, then the Gaussian profile is a better fit for lower heights. There is still a switch to the exponential damping profile at larger heights but as at these heights most of the energy in the $m=1$ mode is transferred into the Alfvén wave, observationally this stage is perhaps not so important for practical seismological purposes. In this paper we have chosen a wider boundary layer as a narrow transition layer does not reproduce the observed rapid/short damping scales (e.g. Goossens et al. 2002; Pascoe et al. 2010).

Acknowledgements. D.J.P. acknowledges financial support from STFC. I.D.M. acknowledges support of a Royal Society University Research Fellowship. The computational work for this paper was carried out on the joint STFC and SFC (SRIF) funded cluster at the University of St Andrews (Scotland, UK).

\section{References}

Arber, T. D., Longbottom, A. W., Gerrard, C. L., \& Milne, A. M. 2001, JCP, 171, 151

Aschwanden, M. J., Fletcher, L., Schrijver, C. J., \& Alexander, D. 1999, ApJ, 520,880

Edwin, P. M., \& Roberts, B. 1983, Sol. Phys., 88, 179

Goossens, M., Hollweg, J. V., \& Sakurai, T. 1992, Sol. Phys., 138, 233

Goossens, M., Andries, J., \& Aschwanden, M. J. 2002, A\&A, 394, L39

Hollweg, J. V., \& Yang, G. 1988, J. Geophys. Res., 93, 5423

Hood, A. W., Brooks, S. J., \& Wright, A. N. 2005, Proc. R. Soc. Lond. A, 461, 237

Mann, I. R., \& Wright, A. N. 1995, J. Geophys. Res., 100, 23677

Mann, I. R., Wright, A. N., \& Hood, A. W. 1997, J. Geophys. Res., 102, 2381

Nakariakov, V. M., Ofman, L., Deluca, E. E., Roberts, B., \& Davila, J. M. 1999, Science, 285, 862

Pascoe, D. J., Wright, A. N., \& De Moortel, I. 2010, ApJ, 711, 990

Pascoe, D. J., Wright, A. N., \& De Moortel, I. 2011a, ApJ, 731, 73

Pascoe, D. J., De Moortel, I., Wright, A. N., \& Hood, A. W. 2011b, ApJ, submitted

Roberts, B., Edwin, P. M., \& Benz, A. O. 1984, ApJ, 279, 857

Ruderman, M. S., \& Roberts, B. 2002, ApJ, 577, 475

Terradas, J., Oliver, R., \& Ballester, J. L. 2006, ApJ, 642, 533

Terradas, J., Goossens, M., \& Verth, G. 2010, A\&A, 524, A23

Tomczyk, S., \& McIntosh, S. W. 2009, ApJ, 697, 1384

Tomczyk, S., McIntosh, S. W., Keil, S. L., et al. 2007, Science, 317, 1192

Van Doorsselaere, T., Andries, J., Poedts, S., \& Goossens, M. 2004, ApJ, 606, 1223

Verth, G., Terradas, J., \& Goossens, M. 2010, ApJ, 718, L102 